PROCEEDINGS OF THE

AMERICAN MATHEMATICAL SOCIETY

Volume 127, Number 11, Pages 3251-3258

S 0002-9939(99)04942-4

Article electronically published on April 27, 1999

\title{
ITERATION OF A CLASS OF HYPERBOLIC MEROMORPHIC FUNCTIONS
}

\author{
P. J. RIPPON AND G. M. STALLARD \\ (Communicated by Mary Rees)
}

Dedicated to Professor Noel Baker on the occasion of his retirement

\begin{abstract}
We look at the class $B_{n}$ which contains those transcendental meromorphic functions $f$ for which the finite singularities of $f^{-n}$ are in a bounded set and prove that, if $f$ belongs to $B_{n}$, then there are no components of the set of normality in which $f^{m n}(z) \rightarrow \infty$ as $m \rightarrow \infty$. We then consider the class $\widehat{B}$ which contains those functions $f$ in $B_{1}$ for which the forward orbits of the singularities of $f^{-1}$ stay away from the Julia set and show (a) that there is a bounded set containing the finite singularities of all the functions $f^{-n}$ and (b) that, for points in the Julia set of $f$, the derivatives $\left(f^{n}\right)^{\prime}$ have exponential-type growth. This justifies the assertion that $\widehat{B}$ is a class of hyperbolic functions.
\end{abstract}

\section{INTRODUCTION}

Let $f$ be a meromorphic function which is not rational of degree less than two, and denote by $f^{n}, n \in \mathbf{N}$, the $n$-th iterate of $f$. The set of normality, $N(f)$, is defined to be the set of points, $z \in \mathbf{C}$, such that $\left(f^{n}\right)_{n \in \mathbf{N}}$ is well-defined, meromorphic and forms a normal family in some neighbourhood of $z$. The complement of $N(f)$ is called the Julia set, $J(f)$, of $f$. An introduction to the properties of these sets can be found in, for example, [3].

We will use the following notation concerning singularities:

$$
\begin{gathered}
S(f)=\left\{z \in \mathbf{C}: z \text { is a singularity of } f^{-1}\right\}, \\
P(f)=\left\{z \in \mathbf{C}: z \text { is a singularity of } f^{-n}, \text { for some } n \in \mathbf{N}\right\} .
\end{gathered}
$$

It was shown by Herring [7, Theorem 7.1.2] that

$$
\left\{z \in \mathbf{C}: z \text { is a singularity of } f^{-n}\right\} \subseteq S_{n}(f)=\bigcup_{j=0}^{n-1} f^{j}\left(S(f) \backslash A_{j}(f)\right),
$$

where

$$
A_{j}(f)=\left\{z \in \mathbf{C}: f^{j} \text { is not analytic at } z\right\}
$$

and that

$$
P(f)=\bigcup_{n=0}^{\infty} S_{n}(f)
$$

Received by the editors September 30, 1997 and, in revised form, January 26, 1998.

1991 Mathematics Subject Classification. Primary 30D05.

(C)1999 American Mathematical Society 
Eremenko and Lyubich [6] investigated the properties of entire functions in the class $B=\{f: f$ is a transcendental meromorphic function with $S(f)$ bounded $\}$.

In Section 2 we look at the properties of functions in the class

$B_{n}=\left\{f: f\right.$ is a transcendental meromorphic function with $S_{n}(f)$ bounded $\}$.

(Note that $B_{1}$ is equal to $B$.) We prove the following result.

Theorem A. If $f \in B_{n}$, then there is no component of $N(f)$ in which $f^{m n}(z) \rightarrow$ $\infty$ as $m \rightarrow \infty$.

Remarks. Our proof is based on ideas of Eremenko and Lyubich [6, Theorem 1] who proved this result in the case when $f$ is entire and $n=1$. The proof of Theorem A given by Bergweiler [3, Theorem 16] uses [3, Lemma 8] which asserts that, if $f \in B$, $p \geq 1$ and 0 is not a pre-image of $\infty$, then there exist a positive constant $R$ and a curve $\Gamma$ connecting 0 to $\infty$ such that $\left|f^{p}(z)\right| \leq R$ for $z \in \Gamma$. Unfortunately, this lemma is not correct, as shown by the counterexample $f(z)=\frac{\tan z}{z}+\frac{\pi}{2}$. Although $f \in B, f^{2}$ is unbounded on each path to $\infty$. The rest of the proof of $[3$, Theorem $16]$ is correct and the reference to [3, Lemma 8$]$ can be successfully replaced by a reference to Lemma 2.1 of this paper.

It follows from Theorem A that, if $f \in B_{n}$, then there can be no periodic cycle $\left\{N_{0}, \ldots, N_{n-1}\right\}$ of components of $N(f)$ with $f^{m n}(z) \rightarrow \infty$ as $m \rightarrow \infty$ in one of the components - such a cycle is known as a cycle of Baker domains or essentially parabolic domains. Thus we have the following Corollary to Theorem A.

Corollary. If $f \in B_{n}$, then $f$ has no Baker domains of period $n$.

Many authors have considered functions in the class

$S=\{f: f$ is a transcendental meromorphic function with $S(f)$ finite $\}$.

It is easy to see that, if $f \in S$, then $f \in \bigcap_{n=1}^{\infty} B_{n}$ and so a special case of the above Corollary is that functions in $S$ have no Baker domains.

In Sections 3 and 4 we consider the iteration of functions in the class

$$
\widehat{B}=\{f: f \in B \quad \text { and } \quad \bar{P}(f) \cap J(f)=\varnothing\},
$$

where $\bar{P}$ denotes closure with respect to the plane. In Section 3 we use Theorem A to prove the following result.

Theorem B. If $f \in \widehat{B}$, then $P(f)$ is bounded.

In Section 4, we use Theorem B together with the results of Section 2 to prove the following result for meromorphic functions which has applications to estimating the Hausdorff dimension of $J(f)$ when $f \in \widehat{B}$; see [8].

Theorem C. If $f \in \widehat{B}$, then there exist $K>1$ and $c>0$ such that

$$
\left|\left(f^{n}\right)^{\prime}(z)\right|>c K^{n} \frac{\left|f^{n}(z)\right|+1}{|z|+1},
$$

for each $z \in J(f) \backslash A_{n}(f), n \in \mathbf{N}$.

If $f$ is rational, then the following conditions are equivalent - see [2, Section 9.7] and $[4$, Section 5.2] and note that, for rational functions, $\bar{P}$ denotes closure in the sphere:

- $\bar{P}(f) \cap J(f)=\varnothing ;$ 
- $\bar{P}(f)$ is a compact subset of $N(f)$;

- $f$ is expanding, in the sense that there exist $K>1$ and $c>0$ such that $\left|\left(f^{n}\right)^{\prime}(z)\right|>c K^{n}$ for each $z \in J(f), n \in \mathbf{N}$.

A rational function with these properties is said to be hyperbolic. For transcendental meromorphic functions, these conditions are no longer equivalent and so it is not clear what the definition of a hyperbolic transcendental meromorphic function should be. In view of Theorems B and C, however, it does seem natural to say that the functions in $\widehat{B}$ are hyperbolic.

\section{Properties of Functions in the Class $B_{n}$}

We use the following notation:

$$
\begin{gathered}
B(z, r)=\{w:|w-z|<r\}, \\
D_{R}=\{z \in \mathbf{C}:|z|>R\} \cup\{\infty\} .
\end{gathered}
$$

The following lemma is probably 'well known'; we include a proof for the sake of completeness.

Lemma 2.1. If $f \in B_{n}$ and $S_{n}(f) \subseteq B(0, R)$, then each component of $f^{-n}\left(D_{R}\right)$ is simply connected in $\mathbf{C}$.

Proof. Let $V$ be a component of $f^{-n}\left(D_{R}\right)$, let $g$ denote a branch of $f^{-n}$ which maps a point of $D_{R}$ into $V$ and let $h$ denote all analytic continuations of $g\left(e^{t}\right)$ to $H=\{t: \operatorname{Re} t>\log R\}$. Then, by the monodromy theorem, $h$ is analytic in $H$ and maps $H$ onto $V$. There are now two cases to consider.

Case A. The function $h$ is univalent in $H$ and hence $h(H)=V$ is simply connected.

Case B. The function $h$ is $2 m \pi i$-periodic in $H$, for some minimal positive integer $m$.

Indeed, if $h$ is not univalent in $H$, then there is some minimal positive integer $m$ for which $h\left(t_{m}\right)=h\left(t_{m}+2 m \pi i\right)$ for some $t_{m} \in H$ and, if $t$ is close to $t_{m}$, then it follows from the open mapping theorem that there exists $t^{\prime}$ close to $t_{m}+2 m \pi i$ with $h(t)=h\left(t^{\prime}\right)$ and hence $t^{\prime}=t+2 m \pi i$. Thus $h$ has period $2 m \pi i$.

In Case B,

$$
h(t)=\varphi\left(e^{t / m}\right), \quad \text { for } t \in H,
$$

where $\varphi(s)=a_{1} s+a_{0}+a_{-1} s^{-1}+\cdots$ is univalent in $\left\{s:|s|>R^{1 / m}\right\}$, and so

$$
f^{n}(z)=\left(\varphi^{-1}(z)\right)^{m}, \quad \text { for } z \in \varphi\left(\left\{s:|s|>R^{1 / m}\right\}\right) .
$$

Now, if $a_{1} \neq 0$, then $\varphi\left(\left\{s:|s|>R^{1 / m}\right\}\right)$ includes a neighbourhood of $\infty$, so

$$
f^{n}(z) \approx a_{1}^{-m} z^{m} \quad \text { as } z \rightarrow \infty .
$$

But (2.1) is impossible because $\infty$ is an essential singularity of $f^{n}$ and not a pole. Thus $a_{1}=0$ and $\varphi$ maps $\left\{s:|s|>R^{1 / m}\right\} \cup\{\infty\}$ onto a simply connected region in $\mathbf{C}$ containing $a_{0}$, and this region is $V$.

We now use Lemma 2.1 to prove the following result.

Lemma 2.2. Let $f$ be a transcendental meromorphic function. There exists $R_{f}$ such that, if $R>R_{f}, S_{n}(f) \subseteq B(0, R)$ and $|z|,\left|f^{n}(z)\right|>R^{2}$, then

$$
\left|\left(f^{n}\right)^{\prime}(z)\right|>\frac{\left|f^{n}(z)\right| \log \left|f^{n}(z)\right|}{16 \pi|z|} .
$$


Proof. First let $c$ be a periodic point of $f$ (see, for example, [3, Theorem 2]) and then take $R_{f}$ so large that $\left|f^{n}(c)\right|<R_{f}$ for each $n \in \mathbf{N}$.

Now suppose that $R>R_{f}, S_{n}(f) \subseteq B(0, R)$ and $|z|,\left|f^{n}(z)\right|>R^{2}$. Let $V$ be the component of $f^{-n}\left(D_{R}\right)$ which contains $z$ and let $g$ denote the branch of $f^{-n}$ that maps $f^{n}(z)$ to $z$. Since $c \notin V$, it follows from Lemma 2.1 that we can choose a branch $L$ of $\log$ so that $L(z-c)$ is analytic on $V$.

If $H=\{t: \operatorname{Re} t>\log R\}$, then

$$
\Phi(t)=L\left(g\left(e^{t}\right)-c\right)
$$

can be analytically continued to $H$, and $\Phi(H)$ does not include any disc of radius greater than $\pi$. Thus, by Bloch's Theorem,

$$
\left|\Phi^{\prime}(t)\right| \leq \frac{\pi}{B(\operatorname{Re} t-\log R)}, \quad \text { for } t \in H
$$

where $B$ denotes Bloch's constant. Hence

$$
\left|\frac{g^{\prime}\left(e^{t}\right) e^{t}}{g\left(e^{t}\right)-c}\right| \leq \frac{\pi}{B(\operatorname{Re} t-\log R)}
$$

where $e^{t}=f^{n}(z)$, and so

$$
\left|\frac{f^{n}(z)}{(z-c)\left(f^{n}\right)^{\prime}(z)}\right|=\left|\frac{g^{\prime}\left(f^{n}(z)\right) f^{n}(z)}{z-c}\right| \leq \frac{\pi}{B\left(\log \left|f^{n}(z)\right|-\log R\right)} .
$$

The lemma follows by using $|z-c| \leq|z|+|c|<2|z|, \log \left|f^{n}(z)\right|>2 \log R$ and $B>\frac{1}{4}$.

Recall that Theorem A states that, if $f \in B_{n}$, then there is no component of $N(f)$ in which $f^{m n}(z) \rightarrow \infty$ as $m \rightarrow \infty$. We are now in a position to give a proof of this result.

Proof of Theorem $A$. If $f \in B_{n}$, then there exists $R>\max \left(e^{16 \pi}, R_{f}\right)$ with $S_{n}(f) \subseteq$ $B(0, R)$. If $N(f)$ has a component $U$ in which $f^{m n}(z) \rightarrow \infty$ as $m \rightarrow \infty$, then there exist $p \in \mathbf{N}, w \in N(f)$ and $r>0$ such that $\bar{B}(w, r) \subset f^{p n}(U)$ and $\left|f^{m n}(z)\right|>R^{2}$, for each $z \in B(w, r), m=0,1,2, \ldots$.

Now let $V_{m}$ be the component of $f^{-n}\left(D_{R}\right)$ in which $U_{m}=f^{n m}(B(w, r))$ lies. Then, taking $c$ to be the same periodic point as in the proof of Lemma 2.2, it follows from Lemma 2.1 that there exists a branch $L_{m}$ of $\log$ for which $L_{m}(z-c)$ is analytic in $V_{m}$. Next, put $T_{m}=L_{m}\left(U_{m}-c\right)$ and $F_{m}(t)=L_{m}\left(f^{n}\left(e^{t}+c\right)-c\right)$, so that $T_{m+1}=F_{m+1}\left(T_{m}\right)$. It follows from (2.2) that, if $t \in T_{m}$, then

$$
\begin{aligned}
\left|F_{m}^{\prime}(t)\right| & =\left|\frac{\left(f^{n}\right)^{\prime}\left(e^{t}+c\right) e^{t}}{f^{n}\left(e^{t}+c\right)-c}\right| \\
& =\left|\frac{\left(f^{n}\right)^{\prime}(z)(z-c)}{f^{n}(z)-c}\right|, \quad \text { where } z=e^{t}+c \in U_{m} \\
& \geq\left|\frac{\left(f^{n}\right)^{\prime}(z)(z-c)}{2 f^{n}(z)}\right| \\
& \geq \frac{B}{2 \pi}\left(\log \left|f^{n}(z)\right|-\log R\right) \\
& \geq \frac{B \log R}{2 \pi} \geq 2
\end{aligned}
$$

and so

$$
\left|\left(F_{m} \circ \cdots \circ F_{1}\right)^{\prime}(t)\right| \geq 2^{m}, \quad \text { for } t \in T_{1}
$$


Thus, by Bloch's Theorem, $T_{m}$ contains a disc of radius $r_{m}$, where $r_{m} \rightarrow \infty$ as $m \rightarrow \infty$. This, however, is impossible since $T_{m} \subseteq L_{m}\left(V_{m}-c\right)$ which contains no disc of radius greater than $\pi$.

\section{Proof of Theorem B}

Recall that Theorem B states that, if $f \in \widehat{B}$, then $P(f)$ is bounded. Let $f \in \widehat{B}$. Since $\bar{S}(f) \subseteq \bar{P}(f)$ and $\bar{P}(f) \cap J(f)=\varnothing$, it follows that $\bar{S}(f) \subseteq N(f)$ and so, since $S(f)$ is bounded, we deduce that $f \in \bigcap_{n=0}^{\infty} B_{n}$. The fact that $S(f) \subseteq N(f)$ also implies that

$$
P(f)=\bigcup_{j=0}^{\infty} f^{j}(S(f)) .
$$

Since $\bar{S}(f)$ is bounded and contained in $N(f)$, there exist $r>0$ and a finite number of points $w_{1}, \ldots, w_{M} \in S(f)$ such that

$$
S(f) \subseteq \bigcup_{i=1}^{M} \bar{B}\left(w_{i}, r\right) \subseteq N(f) .
$$

It follows from (3.1) and (3.2) that

$$
P(f) \subseteq \bigcup_{i=1}^{M} \bigcup_{j=0}^{\infty} f^{j}\left(\bar{B}\left(w_{i}, r\right)\right)
$$

Therefore, for $1 \leq i \leq M$, we let $U_{i}$ denote the component of $N(f)$ which contains $w_{i}$ and consider the possible forward orbits of $U_{i}$.

We first show that $U_{i}$ cannot be a wandering domain. If $U_{i}$ is a wandering domain, that is, $f^{n}\left(U_{i}\right) \cap f^{m}\left(U_{i}\right)=\varnothing$ when $n \neq m$, then there cannot exist a non-constant limit function of $\left\{\left.f^{n}\right|_{U}\right\}$; see, for example, [1, Lemma 2.1]. Since $f \in B_{1}$, it follows from Theorem A that there exist a sequence $\left\{n_{k}\right\}$ and a finite value $a \in \mathbf{C}$ such that $f^{n_{k}}(z) \rightarrow a$ in $U_{i}$ as $n_{k} \rightarrow \infty$. Since $w_{i} \in S(f) \cap U_{i}$, it follows that $a \in \bar{P}(f)$ and, since $f \in \widehat{B}$, this implies that $a \in N(f)$. This, however, is impossible if $U_{i}$ is a wandering domain.

Thus, $U_{i}$ eventually lands in a periodic cycle $\left\{N_{0}, \ldots, N_{n-1}\right\}$ of components of $N(f)$. Since $\bar{P}(f) \cap J(f)=\varnothing$, there are no Siegel discs or Hermann rings and so, for $0 \leq p \leq n-1$, there exists $z_{p} \in \bar{N}_{p}$ with $f^{m n}(z) \rightarrow z_{p}$ locally uniformly in $N_{p}$. Since $f \in \bigcap_{n=0}^{\infty} B_{n}$, it follows from Theorem A that $z_{p} \neq \infty$, for $0 \leq p \leq n-1$, and so $\bigcup_{j=0}^{\infty} f^{j}\left(\bar{B}\left(w_{i}, r\right)\right)$ is bounded. The result now follows from $(3.3)$.

\section{Proof of Theorem C}

The proof of Theorem $\mathrm{C}$ uses results from earlier sections and the following two well known results. The first is Koebe's one-quarter theorem; see for example, [5].

Lemma 4.1. If $f$ is univalent in $B(z, r)$, then

$$
f(B(z, r)) \supset B\left(f(z),\left|f^{\prime}(z)\right| r / 4\right) .
$$

The other result we need is a basic property of Julia sets. Let

$$
\begin{gathered}
O^{-}(w)=\left\{z: f^{n}(z)=w \text { for some } n \in \mathbf{N}\right\}, \\
E(f)=\left\{w: O^{-}(w) \text { is finite }\right\} .
\end{gathered}
$$


If $f$ is meromorphic, then $E(f)$ contains at most two points and we have the following result; see, for example, [3, Section 2].

Lemma 4.2. If $U$ is compact, $U \cap E(f)=\varnothing, z \in J(f)$ and $V$ is an open neighbourhood of $z$, then there exists $N \in \mathbf{N}$ such that, for all $n \geq N$, we have

$$
f^{n}(V) \supset U \text {. }
$$

Theorem $\mathrm{C}$ states that, if $f \in \widehat{B}$, then there exist $K>1$ and $c>0$ such that

$$
\left|\left(f^{n}\right)^{\prime}(z)\right|>c K^{n} \frac{\left|f^{n}(z)\right|+1}{|z|+1},
$$

for each $z \in J(f) \backslash A_{n}(f), n \in \mathbf{N}$.

Let $f \in \widehat{B}$. We know that $\bar{P}(f) \cap J(f)=\varnothing$ and, from Theorem B, that $\bar{P}(f)$ is bounded. Thus there exist $C>1$ and an open set $G$ containing $\bar{P}(f)$, such that

$$
B\left(z, \frac{|z|+1}{C}\right) \cap G=\varnothing
$$

for each $z \in J(f)$.

Since $\bar{P}(f)$ is bounded, it follows from Lemma 2.2 that there exists $R>0$ such that

$$
\left|\left(f^{n}\right)^{\prime}(z)\right|>16 C \frac{\left|f^{n}(z)\right|+1}{|z|+1}, \quad \text { for } n \in \mathbf{N},|z|>R,\left|f^{n}(z)\right|>R .
$$

We now claim that there exists $N_{1} \in \mathbf{N}$ such that

$$
\left|\left(f^{n}\right)^{\prime}(z)\right|>16 C \frac{\left|f^{n}(z)\right|+1}{|z|+1}, \quad \text { for } n \geq N_{1}, z \in\left(J(f) \backslash A_{n}(f)\right) \cap \bar{B}(0, R) .
$$

Otherwise, there exists a sequence of points $z_{n_{k}} \in\left(J(f) \backslash A_{n_{k}}(f)\right) \cap \bar{B}(0, R)$ such that

$$
\left|\left(f^{n_{k}}\right)^{\prime}\left(z_{n_{k}}\right)\right| \leq 16 C \frac{\left|f^{n_{k}}\left(z_{n_{k}}\right)\right|+1}{\left|z_{n_{k}}\right|+1},
$$

with $z_{n_{k}} \rightarrow \alpha \in J(f) \cap \bar{B}(0, R)$ as $n_{k} \rightarrow \infty$.

It follows from (4.1) and Lemma 4.1 that, if $g$ is the branch of $f^{-n_{k}}$ that maps $f^{n_{k}}\left(z_{n_{k}}\right)$ to $z_{n_{k}}$, then

$$
g\left(B\left(f^{n_{k}}\left(z_{n_{k}}\right), \frac{\left|f^{n_{k}}\left(z_{n_{k}}\right)\right|+1}{C}\right)\right) \supseteq B\left(z_{n_{k}}, \frac{\left|z_{n_{k}}\right|+1}{64 C^{2}}\right) .
$$

Thus, for large $n_{k}$,

$$
\begin{aligned}
f^{n_{k}}\left(B\left(\alpha, \frac{1}{100 C^{2}}\right)\right) & \subseteq f^{n_{k}}\left(B\left(z_{n_{k}}, \frac{\left|z_{n_{k}}\right|+1}{64 C^{2}}\right)\right) \\
& \subseteq B\left(f^{n_{k}}\left(z_{n_{k}}\right), \frac{\left|f^{n_{k}}\left(z_{n_{k}}\right)\right|+1}{C}\right)
\end{aligned}
$$

and so, by (4.1),

$$
f^{n}\left(B\left(\alpha, \frac{1}{100 C^{2}}\right)\right) \cap G=\varnothing,
$$

for arbitrarily large values of $n$. Since $\alpha \in J(f)$, this contradicts Lemma 4.2, and hence (4.3) is true. 
Our next claim is that there exists $N_{2} \in \mathbf{N}$ such that, for each $n \geq N_{2}, z \in$ $J(f) \backslash A_{n}(f)$, we have

$$
\left|\left(f^{n}\right)^{\prime}(z)\right|>\frac{1}{8 C} \frac{\left|f^{n}(z)\right|+1}{|z|+1} .
$$

Otherwise, there exists a sequence of points $z_{n_{k}} \in J(f) \backslash A_{n_{k}}(f)$ such that

$$
\left|\left(f^{n_{k}}\right)^{\prime}\left(z_{n_{k}}\right)\right| \leq \frac{1}{8 C} \frac{\left|f^{n_{k}}\left(z_{n_{k}}\right)\right|+1}{\left|z_{n_{k}}\right|+1},
$$

with $z_{n_{k}} \rightarrow \alpha \in J(f)$ or $z_{n_{k}} \rightarrow \infty$ as $n_{k} \rightarrow \infty$.

It follows from (4.1) and Lemma 4.1 that, if $g$ is the branch of $f^{-n_{k}}$ that maps $f^{n_{k}}\left(z_{n_{k}}\right)$ to $z_{n_{k}}$, then

$$
g\left(B\left(f^{n_{k}}\left(z_{n_{k}}\right), \frac{\left|f^{n_{k}}\left(z_{n_{k}}\right)\right|+1}{C}\right)\right) \supseteq B\left(z_{n_{k}}, 2\left(\left|z_{n_{k}}\right|+1\right)\right) \supseteq B\left(0,\left|z_{n_{k}}\right|+1\right) .
$$

Since $z_{n_{k}} \rightarrow \alpha \in J(f)$ or $z_{n_{k}} \rightarrow \infty$ as $n_{k} \rightarrow \infty$, there exist $\beta \in J(f), r>0$ such that, for large values of $n_{k}$,

$$
B(\beta, r) \subseteq B\left(0,\left|z_{n_{k}}\right|+1\right),
$$

and hence

$$
f^{n_{k}}(B(\beta, r)) \subseteq f^{n_{k}}\left(B\left(0,\left|z_{n_{k}}\right|+1\right)\right) \subseteq B\left(f^{n_{k}}\left(z_{n_{k}}\right), \frac{\left|f^{n_{k}}\left(z_{n_{k}}\right)\right|+1}{C}\right) .
$$

Thus, by (4.1),

$$
f^{n}(B(\beta, r)) \cap G=\varnothing,
$$

for arbitrarily large values of $n$. Since $\beta \in J(f)$, this contradicts Lemma 4.2, and hence (4.4) is true.

We now put $N=\max \left(N_{1}, N_{2}\right)$. If $z \in J(f) \backslash A_{2 N+p}(f)$, then it follows from (4.2) and (4.3) that

$$
\left|\left(f^{2 N+p}\right)^{\prime}(z)\right|>16 C \frac{\left|f^{2 N+p}(z)\right|+1}{|z|+1},
$$

for each $p \in \mathbf{N} \cup\{0\}$, provided that either $|z| \leq R$ or $|z|,\left|f^{2 N+p}(z)\right|>R$.

If $z \in J(f) \backslash A_{2 N+p}(f),|z|>R$ and $\left|f^{2 N+p}(z)\right| \leq R$, then either $\left|f^{N}(z)\right| \leq R$ in which case, by (4.3) and (4.4),

$$
\left|\left(f^{2 N+p}\right)^{\prime}(z)\right|>\frac{1}{8 C} \frac{\left|f^{N}(z)\right|+1}{|z|+1} 16 C \frac{\left|f^{2 N+p}(z)\right|+1}{\left|f^{N}(z)\right|+1}=2 \frac{\left|f^{2 N+p}(z)\right|+1}{|z|+1},
$$

or $\left|f^{N}(z)\right|>R$ in which case, by (4.2) and (4.4),

$$
\left|\left(f^{2 N+p}\right)^{\prime}(z)\right|>16 C \frac{\left|f^{N}(z)\right|+1}{|z|+1} \frac{1}{8 C} \frac{\left|f^{2 N+p}(z)\right|+1}{\left|f^{N}(z)\right|+1}=2 \frac{\left|f^{2 N+p}(z)\right|+1}{|z|+1} .
$$

It follows from (4.5), (4.6) and (4.7) that, for each $z \in J(f) \backslash A_{2 N+p}(f), p \in \mathbf{N} \cup\{0\}$, we have

$$
\left|\left(f^{2 N+p}\right)^{\prime}(z)\right|>2 \frac{\left|f^{2 N+p}(z)\right|+1}{|z|+1}
$$


If $n \geq 2 N$, then there exist $m \in \mathbf{N}, 0 \leq p<2 N$ such that $n=m 2 N+p$ and so, if $z \in J(f) \backslash A_{n}(f)$ and $n \geq 4 N$, then it follows from (4.8) that

$$
\left|\left(f^{n}\right)^{\prime}(z)\right|>2^{m} \frac{\left|f^{n}(z)\right|+1}{|z|+1}>\left(2^{\frac{1}{4 N}}\right)^{n} \frac{\left|f^{n}(z)\right|+1}{|z|+1} .
$$

To complete the proof of Theorem $\mathrm{C}$ we need to show that there exist $c_{n}>0$, for $n=1,2, \ldots, 4 N-1$, such that

$$
\left|\left(f^{n}\right)^{\prime}(z)\right|>c_{n} \frac{\left|f^{n}(z)\right|+1}{|z|+1}
$$

for $z \in J(f) \backslash A_{n}(f)$.

If this is not true, then there exist $m \in \mathbf{N}$ and a sequence of points $z_{k} \in$ $J(f) \backslash A_{m}(f)$ such that

$$
\varepsilon_{k}=\frac{\left|\left(f^{m}\right)^{\prime}\left(z_{k}\right)\right|\left(\left|z_{k}\right|+1\right)}{\left|f^{m}\left(z_{k}\right)\right|+1} \rightarrow 0 \quad \text { as } k \rightarrow \infty .
$$

An argument similar to the proof of (4.4) with $\varepsilon_{k}$ instead of $1 /(8 C)$ and $m$ instead of $n_{k}$ now leads to the fact that, for large $k$,

$$
f^{m}\left(B\left(0, \frac{\left|z_{k}\right|+1}{8 C \varepsilon_{k}}\right)\right) \cap G=\varnothing .
$$

Thus $f^{m}(\mathbf{C}) \cap G=\varnothing$, which is a contradiction, and so the proof of Theorem $\mathrm{C}$ is now complete.

\section{ACKNOWLEDGEMENT}

The authors are grateful to Professor I. N. Baker and the referee for several helpful comments.

\section{REFERENCES}

1. I. N. Baker, J. Kotus and L. Yinian, Iterates of meromorphic functions II. J. London Math. Soc. (2) 42 (1990), 267-278. MR 92m:58114

2. A. F. Beardon, Iteration of rational functions, Springer, 1991. MR 92j:30026

3. W. Bergweiler, Iteration of meromorphic functions, Bull. Amer. Math. Soc. 29 (1993), 151188. MR 94c:30033

4. L. Carleson and T. W. Gamelin, Complex dynamics, Springer, 1993. MR 94h:30033

5. P. L. Duren, Univalent functions, Springer, 1983. MR 85j:30034

6. A. E. Eremenko and M. Yu. Lyubich, Dynamical properties of some classes of entire functions, Ann. Inst. Fourier, Grenoble 42 (1992), 989-1020. MR 93k:30034

7. M. E. Herring, An extension of the Julia-Fatou theory of iteration, Ph.D. thesis, University of London, 1994.

8. G. M. Stallard, The Hausdorff dimension of Julia sets of hyperbolic meromorphic functions, To appear in Math. Proc. Camb. Phil. Soc.

Department of Pure Mathematics, The Open University, Walton Hall, Milton Keynes, MK7 6AA, ENGLAND

E-mail address: p.j.rippon@open.ac.uk

E-mail address: g.m.stallard@open.ac.uk 